\title{
BMJ Concordance in diabetic foot ulcer Open infection
}

\author{
E Andrea Nelson, ${ }^{1}$ Michael Ross Backhouse, ${ }^{1}$ Moninder S Bhogal, ${ }^{2}$ \\ Alexandra Wright-Hughes, ${ }^{2}$ Benjamin A Lipsky, ${ }^{3}$ Jane Nixon, ${ }^{2}$ Sarah Brown, ${ }^{2}$ \\ Janine Gray ${ }^{2}$
}

To cite: Nelson EA, Backhouse MR, Bhogal MS, et al. Concordance in diabetic foot ulcer infection. BMJ Open 2013;3:e002370. doi:10.1136/bmjopen-2012002370

\section{- Prepublication history for this paper are available online. To view these files please visit the journal online (http://dx.doi.org/10.1136/ bmjopen-2012-002370).}

Received 19 November 2012 Revised 19 November 2012 Accepted 20 November 2012

This final article is available for use under the terms of the Creative Commons Attribution Non-Commercial 2.0 Licence; see http://bmjopen.bmj.com

\footnotetext{
${ }^{1}$ School of Healthcare, University of Leeds, Leeds, UK

${ }^{2}$ Clinical Trials Research Unit, University of Leeds,

Leeds, UK

${ }^{3}$ University of Washington, Seattle, Washington, USA
}

Correspondence to Professor E Andrea Nelson; E.A.Nelson@leeds.ac.uk

\section{ABSTRACT}

Introduction: Accurate identification of pathogens, rather than colonising bacteria, is a prerequisite for targeted antibiotic therapy to ensure optimal patient outcome in wounds, such as diabetic foot ulcers. Wound swabs are the easiest and most commonly used sampling technique but most published guidelines recommend instead removal of a tissue sample from the wound bed, which is a more complex process. The aim of this study was to assess the concordance between culture results from wound swabs and tissue samples in patients with suspected diabetic foot infection.

Methods and analysis: Patients with a diabetic foot ulcer that is thought to be infected are being recruited from 25 sites across England in a cross-sectional study. The coprimary endpoints for the study are agreement between the two sampling techniques for three microbiological parameters: reported presence of likely isolates identified by the UK Health Protection Agency; resistance of isolates to usual antibiotic agents; and, the number of isolates reported per specimen. Secondary endpoints include appropriateness of the empiric antibiotic therapy prescribed and adverse events. Enrolling 400 patients will provide $80 \%$ power to detect a difference of $3 \%$ in the reported presence of an organism, assuming organism prevalence of $10 \%$, discordance of $5 \%$ and a two-sided test at the $5 \%$ level of significance. Assumed overall prevalence is based on relatively uncommon organisms such as Pseudomonas. We will define acceptable agreement as $\kappa>0.6$.

Ethics and dissemination: Concordance in diabetic foot ulcer infection (CODIFI) will produce robust data to evaluate the two most commonly used sampling techniques employed for patients with a diabetic foot infection. This will help determine whether or not it is important that clinicians take tissue samples rather than swabs in infected ulcers. This study has been approved by the Sheffield NRES Committee (Ref: 11/ $\mathrm{YH} / 0078$ ) and all sites have obtained local approvals prior starting recruitment.

Study registration: NRES Ref: 11/YH/0078, UKCRN ID: 10440, ISRCTN: 52608451

\section{INTRODUCTION}

The global prevalence of diabetes is estimated to be $3 \%$ and predicted to double by

\section{ARTICLE SUMMARY}

Article focus

- Accurate identification of pathogens, rather than colonising bacteria, is a prerequisite for targeted antibiotic therapy to ensure optimal patient outcome in diabetic foot ulcers.

- There is a lack of robust evidence to advise clinicians on the best technique to identify pathogens in diabetic foot ulcers.

- The aim of this study was to assess the concordance between culture results from wound swabs and tissue samples in patients with infected diabetic foot ulcers.

Key messages

- This protocol details the design of the first prospective multicentre study to examine agreement between different wound sampling techniques in patients with diabetic foot ulcers.

- Results from this study will be directly relevant to all clinicians treating patients with diabetic foot ulcers.

Strengths and limitations of this study

- The sample size of 400 patients will provide $80 \%$ power for detecting a difference of $3 \%$ in the reported presence of organisms.

- Recruiting patients from 25 sites across England increases the external validity of results.

- The subset of samples processed using molecular (PCR) techniques will allow the largest comparison of this technology with the conventional plating techniques in diabetic foot ulcers. However, further work will still be required to fully evaluate the role of PCR techniques in the care of diabetic foot ulcers.

2030, largely due to the obesity epidemic. ${ }^{1}$ Among the complications of diabetes, those involving peripheral nerves and arteries predispose to foot complications. These include alterations in foot architecture and mechanics that lead to increased pressure on the plantar surfaces. ${ }^{2}{ }^{3}$ These changes, combined with peripheral sensory and autonomic neuropathy, increase susceptibility to trauma and dry skin fissures. ${ }^{4} 5$ The 
additional presence of peripheral vascular disease impairs wound healing. Thus, it is not surprising that foot ulceration occurs in 15-25\% of diabetic patients during the course of their disease. ${ }^{6} 7$

Diabetic foot ulcers can take many weeks or often months to heal and are known to have a negative impact on patients' functional ability, quality of life, as well as a wider societal impact in terms of reduced work productivity, health costs and financial loss. ${ }^{8-11}$ The combination of immunological perturbations caused by diabetes and an open wound frequently results in clinically apparent infection. At presentation about half of recent onset diabetic foot ulcers are clinically infected. ${ }^{12}$ Diabetic foot infection is thought to be the most common cause of diabetes-related hospital admissions and precedes approximately $80 \%$ of non-traumatic lower-limb amputations. $^{6} 1314$

All chronic wounds have bacteria on their surface, which mostly originate from the normal skin flora, sometimes supplemented by opportunistic colonising bacteria. Thus, the presence of bacteria within a wound is not sufficient to diagnose infection. Infection is instead a clinical diagnosis based on signs and symptoms such as fever, pus, pain or tenderness, erythema, warmth and induration. ${ }^{15}$ These findings suggest a shift in balance in favour of the bacteria within the ulcer over the host's defences, with consequent destruction of host tissue.

When infection is diagnosed, antibiotic therapy is initiated which is usually empirically selected, based on the clinician's understanding of the likely causative organisms. To modify the empiric treatment, if needed, clinicians seek to identify the infective organisms within the wound and their antibiotic susceptibilities through microbiological evaluation. ${ }^{16}$ This may contribute to the clinical diagnosis of infection (by the isolation of virulent organisms likely to be pathogens, or a heavy bacterial load of less virulent organisms). Crucially, it also allows targeted treatment with the most appropriate antibiotic agent for specific organisms, thus reducing the overuse of broad spectrum antimicrobials, which is associated with increasing antibiotic resistance. ${ }^{15-17}$

Currently, culture and sensitivity results typically take several days before they are reported to clinicians, during which time the patient is often treated with broad spectrum antibiotic(s) so as to cover all potential pathogens. Furthermore, it is not clear as to whether clinically relevant changes in the wound flora occur between initial sample and test results being available at reassessment. Quicker techniques for microbiological analyses, such as those based on DNA fingerprinting techniques or the PCR assay, have been shown to be effective in rapidly detecting causative organisms and may help reduce this delay. ${ }^{18}$ In theory, this might lead to earlier tailoring of antimicrobial therapy to infecting organism(s).

The accuracy of the culture information is crucially dependent on an appropriate specimen, that is, one from the infected site that is not contaminated by surrounding normal flora, being sent to the laboratory.
The specimen must also be quickly transported and inoculated on plates to avoid losing pathogens or allowing contaminants to proliferate. Failing to identify a true pathogen (poor test sensitivity) or identifying a coloniser as a pathogen (poor specificity) can each lead to incorrect antibiotic therapy and worse treatment outcomes. Thus, it is important that clinicians use a technique to obtain specimens that will give a valid account of the bacteria present and their number and sensitivity to antibiotics.

\section{Need for a study}

Swabs for taking a culture are almost universally available in healthcare settings and are quick and easy to use. But, they may be susceptible to collecting contaminants and to failing to grow some pathogens. In order to send a specimen from which pathogens are likely to grow, most published expert guidelines recommend obtaining a tissue specimen rather than a swab. ${ }^{19} 20$ Many cite the study by Pellizzer et $a l^{21}$ which reported on 29 diabetic patients who were suspected of severe infection and were neither recently treated with antibiotics, nor hospitalised. The analysis of the study did not report agreement between swab and tissue sample, however, simply the number of bacterial colonies in each sample. Their conclusion that tissue samples are better than swabs can be traced to a comparison of the numbers of isolates in 21 people remaining in the study at 30 day. Furthermore, this selected population does not reflect many of the foot ulcer patients seen in foot clinics, who have often been treated with antibiotics. More recently, Slater et $a l^{22}$ reported the results from swabs and a deeper tissue sample (obtained via needle aspiration), but their study only contained 30 diabetic patients with foot ulcers (in a total sample of 60; other patients had deep abscesses, etc). They found that in 37 patients (62\% of samples), there was a similar profile of organisms isolated from the swab and the deep tissue sample. In 12 patients (20\% of samples), the swab identified more organisms and in 11 patients ( $18 \%$ of samples) the deeper tissue sample identified more organisms. These data were not stratified by the presence or absence of ulcer, or ulcer type (neuropathic/ischaemic) and it is not clear if the results were heterogeneous across tissue damage types or applied to tissue samples.

A systematic review of the diagnosis and management of infection in diabetic foot ulcers ${ }^{23}$ found only one study that evaluated sample acquisition and reported agreement in sufficient detail to allow full analysis. This study, by Bill et $a l^{24}{ }^{24}$ included patients with a variety of wounds: 18 pressure ulcers, 10 diabetic foot ulcers, 5 venous leg ulcers and 5 arterial ulcers. A punch biopsy was taken from the centre of the wound and compared with a wound swab with quantitative analysis. Swabs were not taken from wounds with a bacterial load of less than $10^{5}$ colony-forming units bacteria per gram of tissue. The presence of infection was defined by bacterial load (one million bacteria per gram of tissue). The sensitivity 
for wound swabbing was reported as $79 \%$, meaning that the swab failed to detect approximately one in five wound infections as defined by culture of tissue obtained by punch biopsy. The derived likelihood ratios suggested that the wound swab was not a useful method of identifying infection in chronic wounds. Interpretation of study findings is impeded by small size and heterogeneity in the ulcer population.

It may be that these data are not directly transferable to a clinical diabetic population with a diagnosis of foot ulcer infection (ie, clinical indication for a swab). In addition, there were potential sources of bias, such as the lack of a description of blind test verification and clarity as to whether the same clinical data were available when test results were interpreted as would be available when the test is used in practice. Furthermore, the research question addressed in this proposed study is not about 'diagnosis of infection' (ie, would both tissue sample and swab agree for an arbitrary bacterial load of $>10^{5}$ colonyforming units bacteria per gram of tissue for tissue biopsy and $>10^{5}$ colony-forming units bacteria $/ \mathrm{cm}^{2}$ for swab cultures) as the diagnosis of infection (present or absent) is a clinical decision. People with a bacterial load of $<10^{5}$ colony-forming units bacteria per gram of tissue did not have a wound swab, and it is not clear, therefore, if the swab provides additional information, as suggested by Pellizzer $e t a l^{21}$ Thus, the study by Bill $e t a l^{24}$ cannot conclude that swabs of clinically infected diabetic ulcers do not provide similar information as tissue samples obtained through tissue samples.

\section{Objectives}

Primary objective

The primary objective of this study was to evaluate concordance (agreement) between culture results from wound swabs and tissue samples from the same patient. The coprimary endpoints for the study are agreement between the two techniques for three microbiological parameters.

- Reported presence of isolates likely to be pathogens, as identified by the UK Health Protection Agency (HPA).

- The presence of resistance to antibiotics to which the isolates are usually sensitive among likely isolates as reported by standard techniques.

- The number of bacterial isolates reported per specimen (swab/tissue sample).

\section{Secondary objectives}

Secondary objectives are to

- Compare the proportions of patients for whom empirical antibiotic therapy was 'appropriate', based on culture and sensitivity results of swab or tissue samples, assessed by a blinded clinical panel review (with record of antimicrobial therapy prescribed).

- Compare the number of isolates, reported in both the swab and tissue samples by conventional plating and culture, against molecular techniques (ie, PCR) that identify the nucleic acids of bacteria in the wound.

- Compare rates of adverse effects with the two techniques.

- Compare costs of sampling with the two techniques.

\section{METHODS AND ANALYSIS}

\section{Study design}

This is a multicentre, cross-sectional study involving 400 patients with diabetes presenting with a foot ulcer suspected of being infected who are thought by their clinicians to require antibiotic therapy (see figure 1). Consenting patients will have both a swab and tissue sample taken from their foot ulcer for conventional plating and culture. In addition, 20 patients will be included in a substudy in which a second-swab sample and half of the tissue sample will be processed using molecular (PCR) techniques for comparison with the conventional plating techniques.

\section{Eligibility}

All patients at least 18 years of age with a diabetic foot ulcer that the clinician suspects is infected, either acutely or as a chronic infection, will be screened for enrolment and must meet the eligibility criteria below. A diabetic foot ulcer will be considered to be any open wound on the foot (below the malleoli/ankle) in a patient with a diagnosis of diabetes mellitus. Each participant will undergo an eligibility screen prior to entry and an anonymised log will capture patient demographics along with reasons for not entering the study.

\section{Inclusion criteria}

- The patient has a diagnosis of diabetes (type 1 or type 2).

- The patient is at least 18 years of age at the time of signing the consent form.

- The patient has a suspected ulcer infection with or without bone infection, based on clinical signs and symptoms using Infectious Diseases Society of America/ International Working Group on the Diabetic Foot criteria and the judgement of the investigator.

- The clinical plan is to treat the patient with antibiotics for their infected ulcer.

\section{Exclusion criteria}

- The clinician deems it inappropriate to take a tissue sample or a swab sample for any reason.

- The patient has previously been recruited to the study.

\section{Recruitment and registration}

Centres will be required to have obtained local ethical and management approvals and undertake a site initiation meeting with the Clinical Coordinator prior to the start of recruitment into the study.

Both inpatients and outpatients will be recruited from multidisciplinary primary and secondary care-based foot 
Figure 1 Study flow diagram.

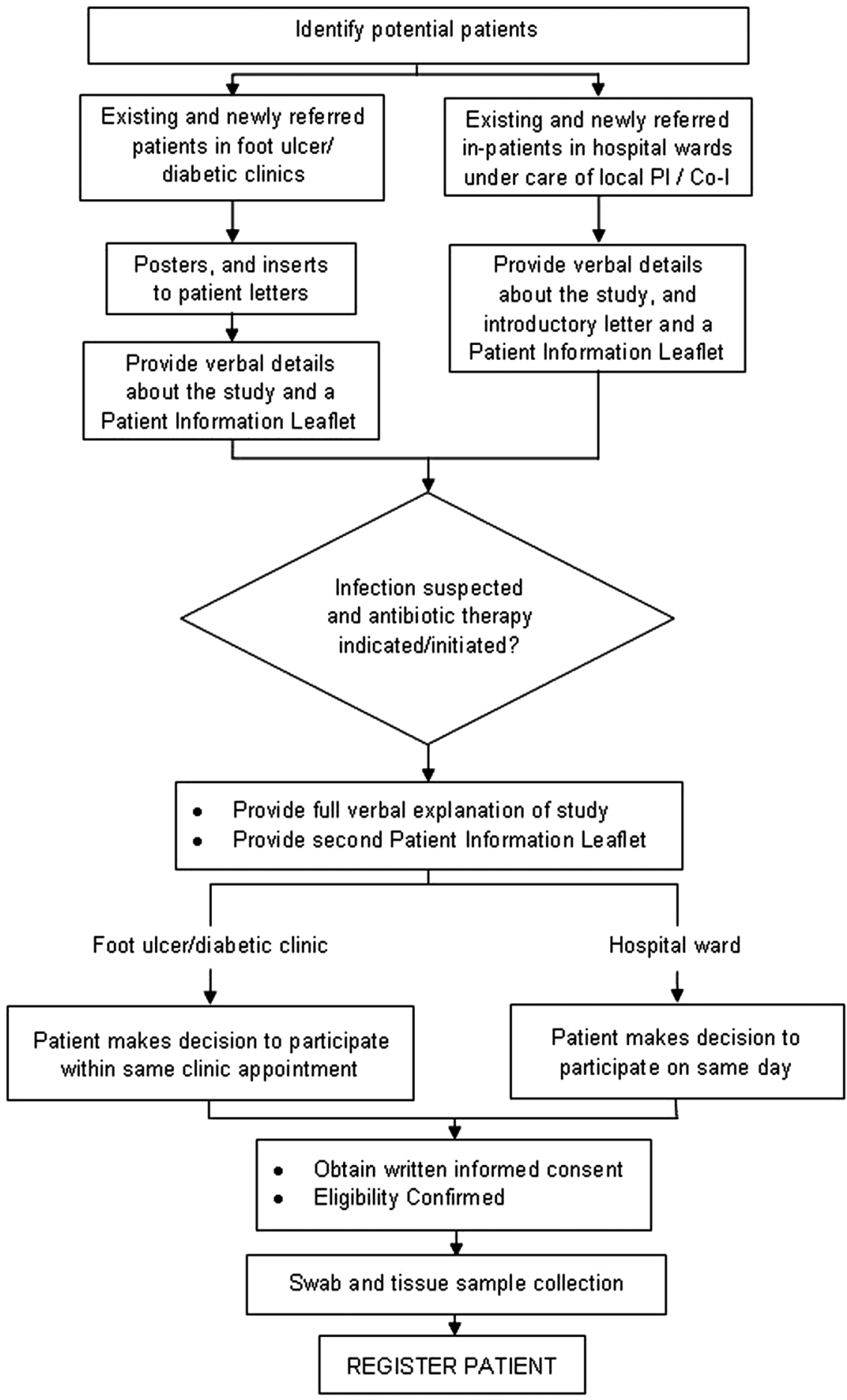

ulcer/diabetic clinics and hospital wards. Potential patients will be provided with a patient information leaflet outlining all aspects of the study, given the chance to read it and ask any questions they may have about the study. Written informed consent will be documented by the patient and member of the local team. Informed written consent will be obtained from all patients prior to entering the study. Patients will be registered via a $24 \mathrm{~h}$ automated telephone registration system that will automatically send confirmation of successful registration through to the site.

\section{Assessments}

Sample acquisition

Clinicians at all centres will be trained to collect samples using the HPA standards ${ }^{25} 26$ as a minimum requirement. In the first instance training will be delivered during the site initiation visit but staff will also be able to access an e-learning package containing a video at any time throughout the study. It is not anticipated that this will substantially alter current swabbing practice as this is a routine procedure with established patterns of practice from the HPA. 
After wound cleansing (using sterile saline and gauze) and debridement (removal of necrotic tissue, foreign material, callus, undermining of the wound edge), a physician, nurse or podiatrist will obtain specimens for aerobic and anaerobic cultures by

- First, using a cotton-tipped swab rubbed over the wound surface to sample superficial wound fluid and tissue debris. The swab will be pressed with sufficient pressure on the wound bed to capture expressed wound fluid, and will be positioned deep in the ulcer to collect from likely infected areas. For the substudy in 20 patients of culturing versus molecular techniques, a wound swab will be collected for conventional techniques and another for molecular analysis.

- Immediately after the cotton swab has been collected, a tissue sample will be removed from the same area of the ulcer bed. This procedure will be done using sterile equipment (forceps, scalpel and scissors) and aseptic technique. It will involve the removal of a small piece of wound tissue at the base of the wound by scraping or scooping using a dermal curette or sterile scalpel blade.

\section{Training of swab and sampling techniques}

Clinicians in the participating sites will participate in a study information session to update their technique for acquiring wound samples. Clinicians will also view an e-learning package that will be developed and issued to all sites, detailing study procedures. This will include video footage of correct methods of obtaining both types of samples.

\section{Sample transport}

Both samples will be placed in transport medium suitable for sustaining both aerobic and anaerobic organisms and promptly delivered to the local medical microbiology laboratory, in accordance with standard practice. A national standard method will be used for collecting and processing samples. ${ }^{25}{ }^{26}$ Both samples will be processed in the same laboratory.

\section{Sample transport for PCR substudy}

For the 20 patients included in the substudy, one swab sample and half of the tissue sample will be sent for molecular analysis, via first class post at ambient temperature to a specialist laboratory. Upon receipt, samples will be stored at $-70^{\circ} \mathrm{C}$ and batches will be defrosted before being processed.

\section{Clinical assessments}

In addition to patient demographics, clinicians will obtain a medical history including information on the patients' diabetes. A detailed foot health history will capture information regarding foot ulcers, including current or proposed antibiotic treatment and dressings. Foot wounds will be categorised and scored by both the PEDIS $^{27}$ and Wagner ${ }^{28}$ ulcer classification systems, along with the Clinical Signs and Symptoms Classification for Infection ${ }^{29}$ to record details of the index ulcer.

A panel will judge the appropriateness of empirical antibiotic therapy against results of both swab and tissue sample findings. The panel will be blind to the source of sample (tissue sample or swab) and to eliminate bias samples will be unpaired and mixed up for judging purposes. Panel members will be asked to comment on the appropriateness of antibiotic selections.

\section{Sample size}

The sample size is based on the outcome reported 'presence or absence of isolate' for the whole sample overall. To be confident that swabs adequately sample wound flora we will assume that the chance corrected agreement between swabs and tissue samples needs to be at least 'good': usually defined as a $\kappa>0.6{ }^{30}$

A sample size of 399 patients will provide $80 \%$ power for detecting a difference of $3 \%$ in the reported presence of any given organism, assuming an overall prevalence for the organism of $10 \%$, a discordance between the swab and tissue samples of $5 \%$, and a two-sided test at the $5 \%$ level of significance. This amount of agreement would provide a $\kappa$ of $\sim 0.7$. This calculation is based on less prevalent organisms, such as Pseudomonas (present in $10 \%$ of samples in ref. ${ }^{21}$ ). Based on these analyses we plan to recruit a total of 400 patients.

The $\kappa$ alone does not convey the distribution of disagreement between swabs and tissue samples and that good overall agreement, with balanced disagreement around the central axis of a table of distributions, would be clinically important if tests were to be regarded as interchangeable. Thus, the total sample size has been based on good agreement between the sample types and reasonably balanced discordance (ie, a small difference due to similar proportions of isolates missed by both swab and tissue sample culture), for clinically important and less prevalent organisms.

The substudy (culture vs molecular techniques) will collect samples from 20 patients to allow an evaluation of the level of agreement and inform a powered, definitive study.

\section{STATISTICAL ANALYSIS}

All tests of statistical significance will be two sided with $\mathrm{p}$ values and $95 \%$ CIs provided, as appropriate. The results will be reported in line with the STARD guidelines. $^{31}$

\section{Patient populations}

All patients registered will be included in the full analysis set. This analysis population will consist of all patients registered to take part in the study, regardless of their adherence to the study protocol or any subsequent discovery of ineligibility. The full analysis set will be used for summarising the patient baseline characteristics. 
The evaluable population will consist of all patients with both evaluable swab and tissue samples. Patients for whom either the swab or tissue samples were not successfully collected or were lost, or for whom the sample results were lost will not be included in this patient population. The evaluable population will be used for the summaries and analyses of all endpoints.

A per-protocol population will be considered if there are a considerable number of protocol violations. The per-protocol population will consist of all registered patients who were not defined protocol violators, which includes eligibility violators and protocol deviators. The per-protocol population will be used for the summaries and analyses of all endpoints.

The safety population will consist of the same patients in the full analysis set, and will be used for summarising adverse events (AEs).

\section{Primary endpoint analysis}

\section{Reported presence of isolates}

For each isolate reported, a cross-tabulation on the semiquantitative extent of growth (none, + to +++ ) will be generated for swab versus tissue samples, by type of diabetic foot ulcer (neuropathic, ischaemic) and overall and weighted $\kappa$ will be reported for all tables. Categories + to +++ will be combined to record any reported presence of the isolate.

The corresponding $2 \times 2$ table will be created and several statistics will be reported: prevalence and bias-adjusted $\kappa$, unadjusted $\kappa$ and overall percentage agreement.

McNemar's test will be used to test for a difference between swab and tissue sampling techniques in the proportion of samples with the reported isolate present, to further investigate the pattern of disagreement.

\section{Summary of isolates reported}

An overall summary of isolates reported will be generated. ${ }^{22}$ Each pair of results (swab and tissue sample) will be coded as follows: swab and tissue sample report all the same isolates; swab reports same isolates as tissue sample plus extra isolates; tissue sample reports same isolates as swab plus extra isolates; tissue sample and swab report different isolates (with or without overlap in some isolates found).

Multinomial logistic regression will model the proportions in each category on type of ulcer (predominantly neuropathic or ischaemic), grade of ulcer, previous antibiotic therapy, antimicrobial dressing, wound duration and centre, to determine whether agreement is influenced by any of the specified covariates. The reference category will be same isolates reported by both tests; estimates of ORs for each covariate will be presented along with $95 \%$ CIs.

\section{Reported presence of antimicrobial resistance} among likely isolates

Methicillin-resistant Staphylococcus aureus, methicillinresistant coagulase-negative staphylococci and vancomycin-resistant Enterococcus species are the three antimicrobial-resistant isolates of most interest. For each of these resistant isolates, $2 \times 2$ tables will be created (presence or absence of resistant isolates) and the following statistics will be reported: prevalence and bias adjusted $\kappa$, unadjusted $\kappa$ and overall percentage agreement.

McNemar's test will be used to test for a difference between swab and tissue sampling techniques in the proportion of samples in which the specified resistant isolate is reported.

For each resistant isolate the following codes will be created: resistant isolate reported by swab but not tissue sample, resistant isolate reported by tissue sample but not swab, swab and tissue sample results agree. Multinomial regression modelling will model these categories on type of ulcer (predominantly neuropathic or ischaemic), grade of ulcer, current antibiotic therapy, antimicrobial dressing, wound duration and centre to determine whether agreement is influenced by any of the specified covariates.

\section{Number of isolates reported}

Summaries (including cross-tabulations) on the number of isolates reported per specimen will be generated for swab versus tissue samples. Samples will be further coded as follows: tissue sample had two or more extra isolates reported, tissue sample had one extra isolate reported, tissue sample and swab had the same number of isolates reported, swab had one extra isolates reported or swab had two more extra isolates reported.

Ordinal logistic regression will model the number of isolates reported per specimen on type of ulcer (ie, predominantly neuropathic or ischaemic), grade of ulcer, previous antibiotic therapy, antimicrobial dressing, wound duration and centre, to determine whether agreement is influenced by any of the specified covariates. The reference category will be the same number of isolates reported by both tests; estimates of ORs for each covariate will be presented along with $95 \%$ CIs.

\section{Secondary endpoint analysis}

Appropriateness of empirical antibiotic therapy

Summaries (including cross-tabulations) will be generated for the codings: no change to therapy required, possible change of therapy following the review of clinical course of the patient, definite change of therapy required. The first two categories will be combined and the resultant $2 \times 2$ table analysed using McNemar's test to determine if one test identifies significantly more patients requiring a definite change in treatment.

Sample pairs will be further coded: results from the swab but not the tissue sample indicates change in therapy, results from the tissue sample but not the swab indicates a change in therapy, swab and tissue sample in agreement on change in therapy. Multinomial regression modelling will model these categories on type of ulcer (predominantly neuropathic or ischaemic), grade of ulcer, previous antibiotic therapy, antimicrobial dressing, 
wound duration and centre to determine whether agreement is influenced by any of the specified covariates.

\section{Number and presence of isolates reported using molecular or culture techniques}

Summaries (including cross-tabulations) on the number of isolates per specimen reported will be generated.

An overall summary of isolates reported using culture and molecular techniques will be generated for both the swab and tissue samples. Each pair of results (from molecular and culture techniques) will be coded as follows: molecular and culture report the same isolates; molecular reports same isolates as culture plus extra isolates; culture reports same isolates as molecular plus extra isolates; the culture and molecular report different isolates (with or without overlap in isolates found).

\section{Adverse events}

Safety analyses will summarise all AEs, serious AEs and related unexpected serious AEs. The number of events and number of patients with events will be summarised.

\section{Sampling costs}

Sampling costs will be summarised for each technique.

\section{DISCUSSION}

Diabetic foot ulcers are highly prevalent and cause considerable morbidity at both the individual and population level. ${ }^{1} 8{ }^{10}$ The combination of a chronic wound and impaired immune defences that may occur in diabetes frequently results in infection; although the sequelae of these complications range in severity, diabetic foot infection precedes $80 \%$ of non-traumatic lower limb amputations. ${ }^{6} 13 \quad 14$ Part of the effective treatment of these infections is providing targeted antibiotic therapy to improve patient outcome and reduce resistance to broad spectrum antibiotics. ${ }^{15-17}$ Despite this, there is a lack of robust evidence to advise clinicians on the best technique to identify pathogens in diabetic foot ulcers.

Concordance in diabetic foot ulcer infection (CODIFI) will generate robust data to evaluate and compare the two most commonly used wound sampling techniques. This holds immediate relevance for all clinicians working with diabetic foot ulcers.

\section{Study status}

CODIFI has received ethical approval from the Sheffield NRES Committee (Ref: 11/YH/0078) and all sites have obtained local approvals prior to commencing recruitment. The study is listed on the UKCRN portfolio (UKCRN ID: 10440) and International Standard Randomised Controlled Trial Number Register (ISRCTN: 52608451). Recruitment opened ahead of schedule in November 2011 and is due to be completed by the end of March 2013.

Acknowledgements We thank members of the study steering committee (Professor J Deeks, Professor R Cooper, Professor R Gadsby, Dr AM Keenan and $C$ Thomas) and the study management group (Professor C Dowson, Professor B Lipsky, Dr E Jude and T Dickie, Dr C Amery, G Sykes, Professor $P$ Vowden, Professor M Edmonds).

Contributors The study was initiated by EAN, who drafted the original study protocol, commented on the draft paper and acts as the chief investigator and guarantor. MRB, MSB, AWH, JN, SB, BL and JG contributed to the study methodology and MRB, MSB, AWH, JN and SB to daily management. MRB drafted the manuscript which all authors commented on and approved the final version. SB was involved in drafting the study protocol and has input into the statistical analysis plan. AWH has drafted and input into the statistical analysis plan.

Funding This project was funded by the NIHR Health Technology Assessment programme (project number 09/75/01) and will be published in full in Health Technology Assessment. The views and opinions expressed herein are those of the authors and do not necessarily reflect those of the HTA programme, NIHR, NHS or the Department of Health.

\section{Competing interests None.}

Ethics approval Sheffield NRES Committee (Ref: 11/YH/0078).

Provenance and peer review Not commissioned; externally peer reviewed.

\section{REFERENCES}

1. Wild SH, Roglic G, Green A, et al. Global prevalence of diabetes: estimates for the year 2000 and projections for 2030. Diabetes care 2004;27:1047-53.

2. Sawacha Z, Spolaor F, Guarneri G, et al. Abnormal muscle activation during gait in diabetes patients with and without neuropathy. Gait \& Posture 2011;35:101-5.

3. Abouaesha F, van Schie CHM, Griffths GD, et al. Plantar tissue thickness is related to peak plantar pressure in the high-risk diabetic foot. Diabetes Care 2001;24:1270.

4. Vinik Al, Maser RE, Mitchell BD, et al. Diabetic autonomic neuropathy. Diabetes Care 2003;26:1553.

5. Richard JL, Lavigne JP, Sotto A. Diabetes and foot infection: more than double trouble. Diabetes Metab Res Rev 2012;28:46-53.

6. Singh N, Armstrong DG, Lipsky BA. Preventing foot ulcers in patients with diabetes. JAMA 2005;293:217.

7. Lavery L, Armstrong D, Wunderlich R, et al. Diabetic foot syndrome: evaluating the prevalence and incidence of foot pathology in Mexican Americans and non-Hispanic whites from a diabetes disease management cohort. Diabetes Care 2003;26:1435.

8. Vileikyte L. Diabetic foot ulcers: a quality of life issue. Diabetes Metab Res Rev 2001;17:246-9.

9. Meijer WG, Trip J, Jaegers SMHJ, et al. Quality of life in patients with diabetic foot ulcers. Disabil Rehabil 2001;23:336-40.

10. Vileikyte L, Rubin RR, Leventhal H. Psychological aspects of diabetic neuropathic foot complications: an overview. Diabetes Metab Res Rev 2004;20(S1):S13-18.

11. Boulton AJM, Kirsner RS, Vileikyte L. Neuropathic diabetic foot ulcers. N Engl J Med 2004;351:48-55.

12. Prompers L, Huijberts $M$, Schaper N, et al. Resource utilisation and costs associated with the treatment of diabetic foot ulcers. Prospective data from the Eurodiale Study. Diabetologia 2008;51:1826-34.

13. Reiber G. The epidemiology of diabetic foot problems. Diabetic Med 1996;13:S6.

14. Armstrong DG, Lavery LA, Quebedeaux TL, et al. Surgical morbidity and the risk of amputation due to infected puncture wounds in diabetic versus nondiabetic adults. J Am Podiatr Med Assoc 1997;87:321-6.

15. Lipsky BA. A report from the international consensus on diagnosing and treating the infected diabetic foot. Diabetes Metab Res Rev 2004;20(S1):S68-77.

16. Lipsky BA, Berendt AR, Deery HG, et al. Diagnosis and treatment of diabetic foot infections. Clin Infect Dis 2004;39:885.

17. Lipsky B. Empirical therapy for diabetic foot infections: are there clinical clues to guide antibiotic selection? Clin Microbiol Infect 2007;13:351-3.

18. Lipsky BA. New developments in diagnosing and treating diabetic foot infections. Diabetes Metab Res Rev 2008;24(S1):S66-71.

19. Armstrong DG, Lipsky BA. Diabetic foot infections: stepwise medical and surgical management. Int Wound J 2004;1:123-32.

20. International Working Group on the Diabetic Foot. International consensus on the diabetic foot (CDROM) (program). Amsterdam: International Diabetes Federation, 2003. 
21. Pellizzer G, Strazzabosco M, Presi S, et al. Deep tissue biopsy vs. superficial swab culture monitoring in the microbiological assessment of limb-threatening diabetic foot infection. Diabet Med $2001 ; 18: 822-7$.

22. Slater R, Lazarovitch T, Boldur I, et al. Swab cultures accurately identify bacterial pathogens in diabetic foot wounds not involving bone. Diabet Med 2004;21:705-9.

23. O'Meara S, Nelson E, Golder S, et al. Systematic review of methods to diagnose infection in foot ulcers in diabetes. Diabet Med 2006;23:341-7.

24. Bill T, Ratliff C, Donovan A, et al. Quantitative swab culture versus tissue biopsy: a comparison in chronic wounds. Ostomy Wound Manag 2001;47:34

25. Health Protection Agency (2009). Investigation of skin superficial and non-surgical wound swabs. National Standard Method BSOP 11 Issue 5. http://www.hpa-standardmethods.org.uk/ pdf_sops.asp
26. Health Protection Agency (2009). Investigation of abscesses and deep-seated wound infections. National Standard Method BSOP 14 Issue 5. http://www.hpa.org.uk/srmd/div_esl_su/pdf_bacteriology.htm

27. Schaper N. Diabetic foot ulcer classification system for research purposes: a progress report on criteria for including patients in research studies. Diabetes Metab Res Rev 2004;20(S1):S90-5.

28. Wagner F Jr. The diabetic foot. Orthopedics 1987;10:163-72: Scale as adapted by $\mathrm{R} \mathrm{G}$. Frykberg, Diabetic foot ulcers: pathogenesis and management. Am Fam Physician 2003;66:9.

29. Gardner S, Frantz R, Troia C, et al. A tool to assess clinical signs and symptoms of localized infection in chronic wounds: development and reliability. Ostomy Wound Manag 2001;47:40.

30. Landis J, Koch $\mathrm{G}$. The measurement of observer agreement for categorical data. Biometrics 1977;33:159.

31. Bossuyt PM, Reitsma JB, Bruns DE, et al. Towards complete and accurate reporting of studies of diagnostic accuracy: the STARD initiative. BMJ 2003;326:41-4. 